NOTICE: this is the author's version of a work that was accepted for publication in Computers \& Geosciences. Changes resulting from the publishing process, such as peer review, editing, corrections, structural formatting, and other quality control mechanisms may not be reflected in this document. Changes may have been made to this work since it was submitted for publication. A definitive version was subsequently published in Computers \& Geosciences, 37 (11), (2011) http://dx.doi.org/10.1016/j.cageo.2011.01.005 
Citation: Hirt C (2011) Mean kernels to improve gravimetric geoid determination based on modified Stokes's integration. Computers \& Geosciences, 37(11):1836-1842, doi:10.1016/j.cageo.2011.01.005.

\title{
Mean kernels to improve gravimetric geoid determination based on modified Stokes's integration
}

\section{Hirt}

Western Australian Centre for Geodesy \& The Institute for Geoscience Research Curtin University of Technology, GPO Box U1987, Perth, WA 6845, Australia Fax:+61 89266 2703; Email: c.hirt@curtin.edu.au

\begin{abstract}
Gravimetric geoid computation is often based on modified Stokes's integration, where Stokes's integral is evaluated with some stochastic or deterministic kernel modification. Accurate numerical evaluation of Stokes's integral requires the modified kernel to be integrated across the area of each discretised grid cell (mean kernel). Evaluating the modified kernel at the centre of the cell (point kernel) is an approximation which may result in larger numerical integration errors near the computation point, where the modified kernel exhibits a strongly nonlinear behaviour. The present study deals with the computation of whole-of-thecell mean values of modified kernels, exemplified here with the Featherstone-Evans-Olliver (1998) kernel modification (Featherstone, W.E., Evans, J.D., Olliver, J.G., 1998. A Meisslmodified Vaníček and Kleusberg kernel to reduce the truncation error in gravimetric geoid computations. Journal of Geodesy 72(3), 154-160). We investigate two approaches (analytical and numerical integration) which are capable of providing accurate mean kernels. The analytical integration approach is based on kernel weighting factors which are used for the conversion of point to mean kernels. For the efficient numerical integration, GaussLegendre Quadrature is applied. The comparison of mean kernels from both approaches shows a satisfactory mutual agreement at the level of $10^{-4}$ and better, which is considered to be sufficient for practical geoid computation requirements. Closed-loop tests based on the EGM2008 geopotential model demonstrate that using mean instead of point kernels reduces numerical integration errors by $\sim 65 \%$. The use of mean kernels is recommended in removecompute-restore geoid determination with the Featherstone-Evans-Olliver (1998) kernel or any other kernel modification under the condition that the kernel changes rapidly across the cells in the neighbourhood of the computation point.
\end{abstract}


Keywords: Geoid determination, Stokes’s integral, modified Stokes’s integration, modified kernel, mean kernel

\section{Introduction}

Many strategies used in gravity field modelling were developed at a time when the "goal for geoid determination was at a precision of a least one order of magnitude less than it is today, i.e., $\sim 10 \mathrm{~cm}$ or worse” (Sjöberg, 2005). For today's geoid and quasigeoid modelling at the centimetre-level and better, it is therefore required to thoroughly assess and - if necessary - correct for approximations that are still inherent in the techniques used.

Regional geoid computations are often based on numerical integration of gravity anomalies using modified Stokes's integration where Stokes's integral is evaluated with some kernel modification (e.g., Featherstone, 2003; Ellmann, 2005). Inevitably, the practical evaluation of Stokes's integral is subject to approximations. This is because Stokes's integral is evaluated by numerical integration of gravity anomalies, given for small surface elements (aka blocks or cells), cf. Heiskanen and Moritz (1967) and Torge (2001). Not only are gravity anomalies required, but also values of the integral kernel which are 'most' representative for the cells (Heiskanen and Moritz, 1967; Vaníček and Krakiwsky, 1986).

Sometimes, the integral kernel is computed at the centre-of-the-cell, but this may be a coarse approximation in the neighbourhood of the computation point, where the kernel changes nonlinearly across the cell (Strang van Hees, 1990). Rigorously, whole-of-the-cell means of the kernel (herein abbreviated to mean kernels) are required. These can be obtained through integration of the kernel over the cell (Vaníček and Krakiwsky, 1986). Using mean instead of centre-of-cell kernels near the computation point may considerably reduce numerical integration errors (Hirt et al. 2011).

For Stokes’s integral and Stokes’s kernel, Strang van Hees (1990) and de Min (1994) have developed approaches to compute estimates of mean kernels across the cell. Recently, Hirt et al. (2011) have combined these approaches, yielding a generalized computation procedure for mean kernels used in Stokes's integral and other geodetic convolution integrals, such as the integrals of Vening-Meinesz and Poisson (Torge, 2001) or Hotine (Hotine, 1969). However, the computation procedures and benefits of mean kernels are generally not or little addressed by scholars for modifications of Stokes's function, such as the 
deterministic modifications of Wong and Gore (1969), Heck and Grüninger (1987), Vaníček and Kleusberg (1987) and Featherstone et al. (1998) and the stochastic modifications of Wenzel (1982) and Sjöberg (1984, 1991). An exception is de Min (1996, p. 169) mentioning the use of cell-mean values for the Wong and Gore (1969) kernel modification.

The aim of the present study is to demonstrate that the use of mean kernel estimates is an important issue for the accurate evaluation of Stokes's integral using modified integration kernels. Section 2 briefly summarizes the basic theory of geoid computation using Stokes's integral, modified Stokes's integration and the Featherstone et al. (1998) kernel as an example for integral kernel modifications. Section 3 then describes and compares one analytical and one numerical approach capable of providing whole-of-the-cell mean values of modified kernels. Results of closed-loop tests are presented in Section 4, revealing the benefits of mean kernels in practice. The Featherstone et al. (1998) kernel has been chosen to serve as example, however, we consider our study representative for other kernel modifications used in practice (e.g., Featherstone, 2003; Ellmann, 2005). The present study is complementary to the paper by Hirt et al. (2011), that discusses the computation of mean kernels for geodetic convolution integrals in general and for the (unmodified) Stokes's integral in particular.

\section{Basic theory}

\subsection{Stokes's integral}

Stokes's integral (aka Stokes's formula) of 1849 allows computation of geoid heights $N$ from gravity anomalies $\Delta g$ which - at least theoretically - are required to be continously given for small cells of size $d \sigma$ covering the whole of the Earth's surface $\sigma$ (Torge, 2001):

$$
N=\frac{R}{4 \pi \gamma} \iint_{\sigma} \Delta g \cdot S(\psi) d \sigma
$$

with $R$ denoting the radius of Earth and $\gamma$ the normal gravity. The term $S(\psi)$ is Stokes's function (aka Stokes's kernel) which is a function of the spherical distance $\psi$ between the computation point $P$ (where the geoid height $N$ is sought) and the data points $Q$ (where gravity anomalies $\Delta g$ are given). In closed-form, Stokes's function reads (Heiskanen and Moritz, 1967): 
$S(\psi)=\frac{1}{\sin (\psi / 2)}-6 \sin (\psi / 2)+1-5 \cos \psi-3 \cos \psi \ln \left[\sin (\psi / 2)+\sin ^{2}(\psi / 2)\right]$.

To contrast with modified kernels in Sections 2.2 and 2.3, $S(\psi)$ is also called unmodified Stokes's kernel. The spherical distance $\psi$ is computed as a function of the spherical coordinates (latitude $\varphi_{P}$ and longitude $\lambda_{P}$ ) of the computation point $P$ and the coordinates $\left(\varphi_{Q}, \lambda_{Q}\right)$ of the data points $Q$ (Strang van Hees 1990, p. 236):

$$
\sin ^{2}\left(\frac{\psi}{2}\right)=\sin ^{2}\left(\frac{\varphi_{P}-\varphi_{Q}}{2}\right)+\sin ^{2}\left(\frac{\lambda_{P}-\lambda_{Q}}{2}\right) \cos \varphi_{P} \cos \varphi_{Q}
$$

Practically, Stokes's integral formula is evaluated numerically by means of summation of products of Stokes's kernel $S(\psi)$ and gravity anomalies $\Delta g$ over small, but finite cells, e.g., of 1 arc min $x 1$ arc min size. The required discretisation of Earth's surface is usually done by means of grid lines along meridians and parallels, giving a subdivision into approximately rectangular cells $q$ (Heiskanen and Moritz, 1967, p. 118). Next, we use $Q$ to denote the centre coordinates of the cells $q$.

Stokes's function $S(\psi)$ is singular for the gravity anomaly of the computation point $P$ (i.e., $\psi=0$ ). The cell centred to the computation point $P$ is also denoted innermost zone (e.g., Featherstone, 2002; Torge, 2001). The contribution of the gravity anomaly $\Delta g_{P}$ of the innermost zone to the geoid height $N$ can be taken into account with approximations given e.g., in Haagmans et al. (1993) or Torge (2001), p. 283.

Evaluating the $S(\psi)$ at the centre of the cells $Q$ is sufficiently accurate beyond some distance, say $1^{\circ}$ from the computation point $P$, while larger numerical integration errors are caused by centre-of-cell kernels in the neighbourhood of the computation point $P$. This is related to the first term of Stokes's function

$$
S^{1 s t}(\psi)=\frac{1}{\sin (\psi / 2)},
$$

which, as dominating component of $S(\psi)$ in the neighbourhood of the computation point $P$, is chiefly responsible for the non-linear across-the-cell-variation of the kernel (see, e.g., Hirt et al., 2011). To accurately evaluate Stokes's integral near the computation point $P$, the kernel 
function needs to be integrated across the cell (Vaníček and Krakiwsky, 1986), which gives whole-of-the-cell mean kernels (Section 3).

\subsection{Modified Stokes’s integration}

It is well known that geoid computation based on Stokes's integral [Eq. (1)] requires the gravity data $\Delta g$ to be provided continuously over the whole of the Earth's surface. This is an impractical requirement, given that the global gravity data coverage (e.g., at 1 arc min resolution) is still incomplete and irregular, thus impeding the direct and accurate use of Stokes’s formula (e.g., Vaníček and Sjöberg, 1991). A commonly applied strategy to circumvent this problem is the remove-compute-restore (RCR) technique together with modified Stokes’s integration (after Vaníček and Sjöberg, 1991, Featherstone et al. 2001):

$$
N=N^{L}+N^{r e s}=N^{L}+\frac{R}{4 \pi \gamma} \iint_{\sigma}\left(\Delta g-\Delta g^{L}\right) \cdot S^{*}(\psi) d \sigma
$$

In the RCR-technique (e.g., Sjöberg, 2005; Featherstone et al., 2001), the observed gravity anomalies $\Delta g$ are reduced ("remove") by the contribution $\Delta g^{L}$ of a global geopotential model (GGM) expanded to spherical harmonic degree $L$, yielding residual anomalies $\Delta g^{\text {res }}=\Delta g-\Delta g^{L}$. These are transformed (“compute”) to the residual geoid undulation $N^{\text {res }}$ of the computation point $P$ using modified Stokes's integration, i.e., evaluation of Stokes's integral in Eq. (5) with some modified kernel $S^{*}(\psi)$. Finally, the GGM geoid contribution $N^{L}$ is added ("restore") to the residual geoid undulation $N^{\text {res }}$. We refer the reader to, e.g., Featherstone et al. (2001) and Torge (2001), p. 271f, for the spherical harmonic expansions to compute $N^{L}$ and $\Delta g^{L}$ from the GGM coefficients. As an important benefit of the RCRtechnique, the computation area may be limited to radius $\psi_{0}$, typically a few degrees, around the computation point $P$ (e.g., Vaníček and Kleusberg, 1987; Featherstone et al., 2001; Torge, 2001, p. 285).

The neglect of gravity data outside the computation area $\left(\psi>\psi_{0}\right)$ results in a truncation error, which is kept manageably small by using a high-degree GGM reference field (e.g., $L=360$ ) in the RCR-approach. In case of using a low-degree GGM reference field (usually well below spherical harmonic degree 360), the truncation error is often estimated and corrected for based on a GGM. This technique, also known as University of New Brunswick approach (e.g., Vaníček and Kleusberg, 1987; Featherstone et al., 2003) is not further discussed here. 
From a range of commonly used kernel modifications (see, e.g., Featherstone, 2003; Ellmann, 2005 for an overview), we select the Featherstone-Evans-Olliver (1998) deterministic modification (herein abbreviated to FEO) to serve as modified kernel $S^{*}(\psi)$ for this study. The Featherstone et al. (1998) modified kernel was used, e.g., in recent RCR-based geoid computations for Australia (Featherstone et al., 2011) and for New Zealand (Claessens et al., 2011).

\subsection{Featherstone et al. (1998) modified kernel}

The FEO modified kernel is based on the Vaníček and Kleusberg (1987) kernel modification and depends on the parameters (i) degree of modification $M$ and (ii) the integration radius (aka integration cap radius or truncation radius) $\psi_{0}$. In a first step, the Wong and Gore (1969) modified kernel $S_{W G}(\psi)$ is computed (Featherstone, 2003)

$$
S_{W G}(\psi)=S(\psi)-\sum_{n=2}^{M} \frac{2 n+1}{n-1} P_{n}(\cos \psi)
$$

where $P_{n}(\cos \psi)$ denotes the Legendre Polynomials (Torge 2001, p.68) and $M$ the degree of modification. In a second step, the Vaníček and Kleusberg (1987) modified kernel $S_{V K}(\psi)$ is calculated

$$
S_{V K}(\psi)=S_{W G}(\psi)-\sum_{n=2}^{M} \frac{2 n+1}{2} t_{n}\left(\psi_{0}\right) P_{n}(\cos \psi)
$$

where the modification coefficients $t_{n}\left(\psi_{0}\right)$ are obtained as solution of a set of $M-1$ linear equations (e.g., Featherstone et al., 2001; Featherstone, 2003)

$$
\sum_{k=2}^{M} \frac{2 k+1}{2} t_{n}\left(\psi_{0}\right) e_{n k}\left(\psi_{0}\right)=Q_{n}\left(\psi_{0}\right)-\sum_{k=2}^{M} \frac{2 k+1}{k-1} e_{n k}\left(\psi_{0}\right) .
$$

The quantities $e_{n k}\left(\psi_{0}\right)$ and $Q_{n}\left(\psi_{0}\right)$ (see Featherstone et al. 2001)

$$
\begin{aligned}
& e_{n k}\left(\psi_{0}\right)=\int_{\psi_{0}}^{\pi} P_{k}(\cos \psi) P_{n}(\cos \psi) \sin \psi d \psi \\
& Q_{n}\left(\psi_{0}\right)=\int_{\psi_{0}}^{\pi} S(\psi) P_{n}(\cos \psi) \sin \psi d \psi
\end{aligned}
$$


are computed using the algorithms by Paul (1973). In a third step, the FEO modified kernel $S_{F E O}(\psi)$ is obtained by forcing the Vaníček and Kleusberg kernel to zero at the integration radius $\psi_{0}$ (Featherstone et al. 1998, p. 157):

$$
S_{F E O}(\psi)=S_{V K}(\psi)-S_{V K}\left(\psi_{0}\right)
$$

Eqs. (7) and (11) are valid for $0<\psi \leq \psi_{0}$. The modified kernel function $S_{F E O}(\psi)$ can be computed with the publicly available software modkern.f (Featherstone, 2003), allowing the reader to replicate the computation of the modified kernel.

The degree of modification $M$ often ranges between 2 and 360 and the integration radius $\psi_{0}$ usually amounts to a few degrees (e.g., Vaníček and Featherstone, 1998; Featherstone et al., 2011; Claessens et al., 2011). Importantly, the spherical harmonic degree $L$ of the GGM reference field and degree of modification $M$ do not need to be equal (e.g., Featherstone et al., 2001). For instance, in practical RCR-based geoid computations using ultra-high-degree GGM reference fields $(L=2160)$, the kernel parameters $M=20 . .60$ and $\psi_{0}=2.5^{\circ} . .5^{\circ}$ have been found to produce accurate geoid heights (e.g., Featherstone et al., 2011; Claessens et al., 2011). This serves as a justification for using the FEO kernel modification with $M=40$ and $\psi_{0}=5^{\circ}$ in the numerical tests of our study. To speed up the geoid computation using modified kernels, it is beneficial to establish a look-up-table (LUT) of pre-calculated kernel values $\left(0<\psi \leq \psi_{0}\right)$ which are cubically interpolated.

The FEO kernel modification $S_{F E O}(\psi)$ (with $L=40$ and $\psi_{0}=5^{\circ}$ ), the [unmodified] Stokes's kernel $S(\psi)$ and the first term of Stokes's function $S^{1 s t}(\psi)$ are shown together in Fig. 1 (left), illustrating the relationship among the functions. For small $\psi$-values, say less than $\sim 0.5-1^{\circ}$, the FEO kernel modification is dominated by the first term of Stokes's function $S^{1 s t}(\psi)$, however not as strongly as the unmodified Stokes’s kernel $S(\psi)$ (Fig. 1 right).

Please place Figure 1 near here

\section{Computation of mean kernels}

Whole-of-the-cell mean values of modified kernels can be obtained either through analytical or numerical integration. In the analytical integration approach, a planar approximation of the 
first term of Stokes's function is integrated over the area of the cell, serving as an aid in computing so-called kernel weighting factors (e.g., Strang van Hees, 1990; Featherstone and Olliver, 1997; Hirt et al., 2011). These are conversion factors allowing transformation from point to mean kernel values (of Stokes's function or modified versions thereof). The numerical integration approach used here is based on Gauss-Legendre Quadrature (e.g., Abramowitz and Stegun, 1972; Hamming, 1986). Both approaches are detailed and compared next.

\subsection{Analytical integration}

The method presented here is a hybrid of the analytical integration approach developed for the unmodified Stokes's kernel by Strang van Hees (1990) and de Min (1994). Strang van Hees (1990) introduced kernel weighting factors $W$ allowing transformation from point to mean kernels. He derived mean Stokes's kernels from a one-dimensional analytical integration assuming square-shaped cells, which limits the accuracy of his method in practice (Hirt et al., 2011). Without using kernel weighting factors, de Min (1994) applied twodimensional analytical integration (2D-AI) of Stokes's function, taking into account the usually rectangular shape of cells. Combining the kernel weighting factor concept with the 2D-AI is a suitable approach to provide analytically mean values of modified kernels.

We start by introducing rectangular coordinates $(x, y)$ to describe the distance between the computation point $\left(\varphi_{P}, \lambda_{P}\right)$ and centre of the cell under evaluation $\left(\varphi_{Q}, \lambda_{Q}\right)$ in planar approximation:

$$
\begin{aligned}
& x=\left(\lambda_{Q}-\lambda_{P}\right) \cos \varphi_{Q}, \\
& y=\varphi_{Q}-\varphi_{P} .
\end{aligned}
$$

Planar approximation of the first term of Stokes's function $S^{1 s t}(\psi) \approx 2 / \psi$ and planar approximation of the distance $\psi \approx \sqrt{x^{2}+y^{2}}$ (see Torge, 2001, p. 287) gives Stokes's kernel in planar approximation (e.g., de Min 1994):

$$
K(x, y)=\frac{2}{\sqrt{x^{2}+y^{2}}} .
$$

For a given cell of size $\Delta \varphi \times \Delta \lambda$ (e.g., 1 arc min $\times 1$ arc min), bounded by corner coordinates $\left(x_{1}, y_{1}\right)$ and $\left(x_{2}, y_{2}\right)$ 


$$
\begin{aligned}
& x_{1}=\left(\lambda_{Q}-\lambda_{P}-\frac{\Delta \lambda}{2}\right) \cos \varphi_{Q}, y_{1}=\varphi_{Q}-\varphi_{P}-\frac{\Delta \varphi}{2}, \\
& x_{2}=\left(\lambda_{Q}-\lambda_{P}+\frac{\Delta \lambda}{2}\right) \cos \varphi_{Q}, y_{2}=\varphi_{Q}-\varphi_{P}+\frac{\Delta \varphi}{2},
\end{aligned}
$$

the whole-of-the-cell mean kernel $\bar{K}\left(x_{1}, y_{1}, x_{2}, y_{2}\right)$ in planar approximation is obtained from two-dimensional analytical integration (after Vaníček and Krakiwsky, 1986; de Min, 1994):

$$
\bar{K}\left(x_{1}, y_{1}, x_{2}, y_{2}\right)=\frac{1}{a} \int_{x=x_{1}}^{x_{2}} \int_{y=y_{1}}^{y_{2}} K(x, y) d x d y
$$

where $a=\left(x_{2}-x_{1}\right)\left(y_{2}-y_{1}\right)$ denotes the area of the cell. Eq. (15) can be solved analytically (de Min, 1994):

$$
\begin{aligned}
& \bar{K}\left(x_{1}, y_{1}, x_{2}, y_{2}\right)=\left.\left.\frac{1}{a}|| F(x, y)\right|_{y_{1}} ^{y_{2}}\right|_{x_{1}} ^{x_{2}}= \\
& \frac{1}{a}\left[F\left(x_{1}, y_{1}\right)-F\left(x_{1}, y_{2}\right)-F\left(x_{2}, y_{1}\right)+F\left(x_{2}, y_{2}\right)\right]
\end{aligned}
$$

using the antiderivative (de Min, 1994)

$$
F(x, y)=2\left[x \ln \left(y+\sqrt{x^{2}+y^{2}}\right)+y \ln \left(x+\sqrt{x^{2}+y^{2}}\right)\right] .
$$

Comparing the mean value $\bar{K}\left(x_{1}, y_{1}, x_{2}, y_{2}\right)$ with the point (centre-of-cell) value $K(x, y)$ (both in planar approximation) gives the kernel weighting factor (Hirt et al., 2011)

$$
W\left(x_{1}, y_{1}, x_{2}, y_{2}\right)=\bar{K}\left(x_{1}, y_{1}, x_{2}, y_{2}\right) / K(x, y)
$$

which is adapted here to transform point values of FEO kernels $S_{F E O}$ to mean values $\bar{S}_{F E O}^{2 D A I}$ for a given cell $\left(x_{1}, y_{1}, x_{2}, y_{2}\right)$ :

$$
\bar{S}_{F E O}^{2 D A I} \approx W S_{F E O} .
$$

Because the first term of Stokes's function $S^{1 s t}(\psi)$ 'governs' the modified kernel $S_{F E O}$ near the computation point (say well within $1^{\circ}$ radius, see also Fig. 1), the kernel weighting factors $\bar{S}_{F E O}^{2 D A I} / S_{F E O}$ and $\bar{K} / K$ have very similar values. This makes the transformation in Eq. (19) possible. A numerical confirmation of Eq. (19) is given in Section 3.3. It should be noted 
that the planar approximations $\bar{K}$ or $K$ of Stokes's kernel are not used as a substitute of the modified kernel $S_{F E O}$. The direct use of the planar approximations (in place of FEO mean kernels $\bar{S}_{F E O}^{2 D A I}$ ) would significantly worsen the accuracy of Stokes’s integration.

\subsection{Numerical integration}

Gauss-Legendre Quadrature (GLQ) (e.g., Stark, 1970; Abramowitz and Stegun, 1972; Hamming, 1986; Alberts and Klees, 2004; Wild-Pfeiffer, 2008) is a very efficient integration technique for the numerical computation of mean kernel estimates. The basic idea is to evaluate the kernel function at carefully selected points (being a function of the Legendre Polynomials), located within the area of the cell $q$. The GLQ numerical mean kernel estimate is obtained as weighted average of the point kernel evaluations. For a given cell $q$, bounded by coordinates $\lambda_{1}, \lambda_{2}, \varphi_{1}, \varphi_{2}$, the GLQ numerical whole-of-the-cell mean value $\bar{S}_{F E O}^{G L Q}$ of the FEO kernel is obtained through $n^{2}$ evaluations of the modified kernel function $S_{\text {FEO }}(\psi$ ) (see also Hirt et al., 2011):

$\bar{S}_{F E O}^{G L Q}=\frac{1}{w_{i} w_{j}} \sum_{i=1}^{n} \sum_{j=1}^{n} w_{i} w_{j} S_{F E O}\left(\psi\left(\varphi_{P}, \lambda_{P}, \varphi_{Q i}, \lambda_{Q j}\right)\right)$

where $n$ is the quadrature degree, $w_{i}, w_{j}$ are the weights (not to confuse with kernel weighting factors $W$ from Section 3.1) and $\varphi_{Q i}, \lambda_{Q j}$ are the coordinates of the evaluation points.. The evaluation points $\varphi_{\mathrm{Qi}}, \lambda_{\mathrm{Qj}}$ are located within the area of cell $q$ (i.e., $\lambda_{1}<\lambda_{\mathrm{Qj}}<\lambda_{2}$ and $\left.\varphi_{1}<\varphi_{\mathrm{Qi}}<\varphi_{2}\right)$ and obtained from (Hirt et al., 2011)

$$
\begin{aligned}
& \lambda_{Q i}=\frac{\lambda_{2}-\lambda_{1}}{2} x_{i}+\frac{\lambda_{2}+\lambda_{1}}{2} \\
& \varphi_{Q j}=\frac{\varphi_{2}-\varphi_{1}}{2} x_{j}+\frac{\varphi_{2}+\varphi_{1}}{2}
\end{aligned}
$$

where $x_{i}\left(x_{j}\right)$ is the $i$-th $(j-$ th) zero crossing of the $n$-th Legendre polynomial (e.g., Abramowitz and Stegun, 1972). The weights $w_{i}, w_{j}$ are computed from (Abramowitz and Stegun, 1972, p. 887; Hirt et al., 2011): 


$$
\begin{aligned}
& w_{i}=\frac{\lambda_{2}-\lambda_{1}}{\left(1-x_{i}^{2}\right)\left[P_{n}^{\prime}\left(x_{i}\right)\right]^{2}} \\
& w_{j}=\frac{\varphi_{2}-\varphi_{1}}{\left(1-x_{j}^{2}\right)\left[P_{n}^{\prime}\left(x_{j}\right)\right]^{2}}
\end{aligned}
$$

with $P_{n}^{\prime}$ being the derivative of the $n$-th Legendre polynomial, evaluated at $x_{i}$ and $x_{j}$, respectively. The computation of the coordinates of the evaluation points $\varphi_{Q i}, \lambda_{Q j}$ and the weights $w_{i}, w_{j}$ can be accomplished with computer code from the Numerical Recipes (Press et al., 2002) or other mathematical software libraries. Naturally, the described approach can be used to compute mean kernel estimates of any other kernel modification.

For the practical application of GLQ, it is useful to introduce some convergence threshold. Given that the geoid signal in RCR-based geoid computation rarely exceeds an order of a few metres, a threshold of $\varepsilon=10^{-4}$ would result in an acceptable uncertainty well below the millimetre level. To investigate the quadrature degree $n$ required, Eq. (20) was evaluated as a function of $n$, ranging from 1 to 20, and compared against a 'reference value' $\bar{S}_{F E O}^{G L Q}(r e f)$ from $n=1000$. The convergence error

$$
\varepsilon=\frac{\bar{S}_{F E O}^{G L Q}(n)-\bar{S}_{F E O}^{G L Q}(r e f)}{\bar{S}_{F E O}^{G L Q}(r e f)}
$$

is shown in Fig. 2 for cells located 1, 5 and 30 arc min North of the innermost zone (cell size is $1 \operatorname{arc} \min x 1$ arc min and latitude of the computation point $P$ is $-35^{\circ}$ ). For cells directly adjoining the innermost zone, a degree 3 quadrature (i.e., 9 evaluation points) is required to provide GLQ numerical mean kernels accurate to $10^{-4}$. In 5 arc min distance from the computation point, 4 evaluation points per cell are sufficient to meet the $10^{-4}$ criterion, whereas the point kernel (equivalent to quadrature degree 1 with the cell centre $Q$ as only evaluation point) is yet good enough in 30 arc min distance and beyond. For higher quadrature degrees, say $n=5$ and higher, convergence errors are at the level of $\sim 10^{-8}$ (Fig. 2). This very small level of uncertainty would be more than sufficient for any practical application of mean kernels in modified Stokes's integration. The results of this convergence experiment demonstrate that GLQ is a very efficient numerical integration technique for the accurate computation of whole-of-the-cell mean values of modified kernels. 
Please place Figure 2 near here

\subsection{Comparisons}

To evaluate the 2D-AI analytical and GLQ numerical computation approach, we computed FEO kernel values for a grid of 1 arc min $\mathrm{x} 1$ arc min cells over Australia. Fig. 3A shows the FEO point kernel matrix (with the computation point $P$ as the centre). The percentage differences between the 2D-AI mean kernel $\bar{S}_{F F O}^{2 D A I}$ and point kernel $S_{F F O}$ are shown in Fig. 3B and those between the GLQ numerical mean $\bar{S}_{F E O}^{G L Q}$ and point kernel $S_{F E O}$ in Fig. 3C. Both approaches exhibit very similar difference patterns with maximum differences of $\sim 5 \%$ between FEO mean and point kernels for cells adjoining the computation point. This demonstrates across-the-cell variations of the FEO mean kernel for small $\psi$-values. Fig. 3D shows the percentage differences between 2D-AI and GLQ mean kernels. The differences are largest adjacent to the innermost zone (about $0.02 \%$ ) and otherwise below or well below the level of $10^{-4}$. This good mutual agreement is considered a sufficient validation of both mean kernel computation strategies. Further to this, the small discrepancies in Fig. 3D implicitly confirm the validity of Eq. (19). As a conclusion, the comparisons demonstrate the capability of both approaches to accurately compute FEO mean kernel estimates.

With both approaches, it will be usually sufficient to restrict the mean kernel computation to a zone of $\sim 30$ arc min radius around the innermost zone (cf. Fig. 2). The increase in computation time (with respect to point kernels) is below $\sim 10 \%$ for both methods, which should be well acceptable in practical applications.

Please place Figure 3 near here

\section{Numerical closed-loop tests}

Closed-loop tests were used to compare and evaluate the performance of FEO analytical/numerical mean and point kernels in RCR-based geoid computation. The recent 
high-resolution EGM2008 global gravity model (Pavlis et al., 2008) served as source to generate self-consistent pairs of gravity anomalies $\Delta g_{E G M 2008}$ and geoid heights $N_{E G M 2008}$ using the state-of-the-art harmonic_synth spherical harmonic synthesis software (Holmes and Pavlis 2008). Transformation of gravity anomalies $\Delta g_{E G M 2008}$ to geoid heights $N\left(\Delta g_{E G M 2008}\right)$ using different FEO kernel variants and subsequent comparision against the 'error free' geoid heights $N_{E G M 2008}$ allows assessment of the mean and point kernel performance.

Australia, Europe and the Himalaya mountains were chosen as test areas to cover different latitudes and signal strengths of gravity field features. The boundaries of the computation areas and signal strengths of the geoid heights are found in the captions of Tables 1-3. For any of the three test areas, the EGM2008 gravity anomalies $\Delta g_{E G M 2008}$ and geoid heights were synthesized on 1 arc min grids in the spectral band 361..2190. This band was chosen because it 'replicates' the frequently used RCR-scheme with a degree $L=360$ GGM reference field removed. Given that a degree 2190 spherical harmonic expansion represents the geoid signal almost completely [the missing portion, known as omission error, is globally estimated to be $\sim 4 \mathrm{~cm}$, cf. Jekeli et al. (2009)], the chosen spectral band is considered a realistic simulation of the RCR-approach.

The FFT1Dmod2010 software of the Western Australian Centre of Geodesy was applied to evaluate Stokes’s integral [Eq. (5)], using the FEO modified kernel [Eq. (9)] with parameters $M=40$ and $\psi_{0}=5^{\circ}$. This software is based on the one-dimensional Fast Fourier Transform technique (Haagmans et al. 1993) that allows very efficient numerical evaluation of Stokes's integral, see also Featherstone et al. (2001). To allow Stokes's integral to be correctly evaluated over the whole cap radius, also near the edges of our test area, the EGM2008 gravity grids were extended by an auxilliary $5^{\circ}$ boundary strip. The descriptive statistics of the closed-loop differences $N_{E G M 2008}-N\left(\Delta g_{E G M 2008}\right)$ show an almost identical performance of the FEO 2D-AI analytical and FEO GLQ numerical mean kernel (Tables 1-3). The practical equivalence of both variants in our tests is as expected, given the $\sim 10^{-4}$ mutual agreement between both mean kernel computation approaches found in Sect. 3.3.

In any of the test areas, FEO mean kernels produce RMS errors which are factor $\sim 2.5$ to 3 lower than those of FEO point kernels (Tables 1-3). Likewise, it is seen that the maximum discrepancies drop from the $\mathrm{cm}$ to the mm-level (Australia) from $\sim 2 \mathrm{~cm}$ to the sub-cm-level 
(Europe) and from 3-4 $\mathrm{cm}$ to the $1 \mathrm{~cm}$-level (Himalaya Mountains). This suggests to use mean kernel estimates in Stokes’ numerical integration with modified kernels.

A detail display of the FEO mean and point kernel performance is given from the closed-loop differences $N_{E G M 2008}-N\left(\Delta g_{E G M 2008}\right)$ along parallel of $-35^{\circ}$ (test area Australia) in Fig. 4, showing the larger numerical integration errors in case of point kernels. Finally, Fig. 5 shows the closed-loop differences $N_{E G M 2008}-N\left(\Delta g_{E G M 2008}\right)$ for the Himalaya test area, illustrating the error patterns caused by using point kernels instead of mean kernels.

Please place Figure 4 near here

Please place Figure 5 near here

\section{Conclusions and recommendations}

The present study has investigated the computation of whole-of-the-cell means for the Featherstone-Evans-Olliver (1998) kernel, serving as an example of modified kernels used in gravimetric geoid determination based on Stokes's integration. We have described (i) the analytical approach to compute FEO mean kernels adapting the concept of kernel weighting factors and (ii) the numerical approach which is based on Gauss-Legendre-Quadrature. The comparison of both methods showed a satisfactory mutual consistency on the level of $\sim 10^{-4}$ and better.

The performance of our mean kernel approaches was compared against centre-of-cell (point) kernels by means of closed-loop tests based on self-consistent sets of gravity anomalies and geoid heights from EGM2008 in the spectral band 361..2190. Any of the test demonstrated that whole-of-the-cell mean values of the FEO kernel modification reduce the numerical integration errors by $\sim 65 \%$. In many cases, maximum closed-loop errors were reduced from some $\mathrm{cm}$ to the sub-cm level, which is a relevant improvement for accurate geoid determination.

Our study demonstrates that mean kernels should be used instead of point kernels to accurately evaluate Stokes's integral with kernel modifications. We have exemplified this with Featherstone-Evans-Olliver (1998) kernel modification, one of many kernel modifications used in practical geoid computations. Our findings are considered 
representative for any other kernel modification under the condition that the modified kernel rapidly varies across the cell in the neighbourhood of the innermost zone. Most of the modified kernels will meet this condition. As a general conclusion, using mean kernels in modified Stokes’s integration considerably reduces numerical integration errors.

\section{Acknowledgements}

The author would like to thank the Australian Research Council for funding through discovery project grant DP0663020. Parts of the computations were performed using the Western Australian iVEC high-performance computational facility. The author is grateful to Will Featherstone for his continuous support and provision of a basis version of the 1DFFT software. Sincere thanks go to Sten Claessens and Will Featherstone for productive discussions and to Robert Kingdon for helpful comments on the manuscript. Fig. 5 was created using the Generic Mapping Tools (Wessel and Smith, 1998). This is the Institute for Geoscience Research (TIGeR) publication number XX.

\section{References}

Abramowitz, M.A., Stegun, I.A., 1972. Handbook of mathematical functions. Dover Publications, New York, 1046 pages.

Alberts, B., Klees, R., 2004. A comparison of methods for the inversion of airborne gravity data. J Geod 78(1):55-65. DOI 10.1007/s00190-003-0366-x.

Claessens, S.J., Hirt, C., Amos, M.J., Featherstone, W.E., Kirby, J.F., 2011. The NZGEOID09 New Zealand quasigeoid model. Survey Review, 43(319): 2-15, doi: 10.1179/003962610X12747001420780.

de Min, E., 1994. On the numerical evaluation of Stokes' integral. International Geoid Service Bulletin 3, 41-46.

de Min, E., 1996. De Geoide voor Nederland. Nederlandse Commissie voor Geodesie Publikatie 34 (Dutch Geodetic Commission Publication No 34), Delft.

Ellmann, A., 2005. Computation of three stochastic modifications of Stokes's formula for regional geoid determination. Computers and Geosciences 31, 742-755, doi:10.1016/j.cageo.2005.01.008.

Featherstone W.E., Olliver J.G., 1997. A method to validate gravimetric geoid computation software based on Stokes's integral. Journal of Geodesy 71(9), 571-576, doi: 10.1007/s001900050125.

Featherstone, W.E., Evans, J.D., Olliver, J.G., 1998. A Meissl-modified Vaníček and Kleusberg kernel to reduce the truncation error in gravimetric geoid computations. Journal of Geodesy 72(3), 154-160.

Featherstone, W.E., Kirby, J.F., Kearsley, A.H.W., Gilliland, J.R., Johnston, G.M., Steed, J., Forsberg, R., Sideris, M.G., 2001. The AUSGeoid98 geoid model of Australia: data 
treatment, computations and comparisons with GPS-levelling data. Journal of Geodesy 75(5-6), 313-330, doi:10.1007/s001900100177.

Featherstone, W.E., 2002. A simple method to improve the geoid from a global geopotential model (or coarse geoid estimation using only the innermost zone contribution of Stokes’s formula). International Geoid Service Bulletin, 12, 79-88.

Featherstone, W.E., 2003. Software for computing five existing types of deterministically modified integration kernel for gravimetric geoid determination. Computers and Geosciences 29(2), 183-193, doi: 10.1016/S0098-3004(02)00074-2.

Featherstone, W.E., Holmes S.A., Kirby J.F., Kuhn M., 2003. Comparison of removecompute-restore and University of New Brunswick techniques to geoid determination over Australia, and inclusion of Wiener-type filters in reference field contribution. Journal of Surveying Engineering 130(1), 40-47, doi: 10.1061/ ASCE!07339453 2004!130:1 40.

Featherstone, W.E., Kirby, J.F., Hirt, C., Filmer, M.S., Claessens, S.J., Brown, N., Hu, G., Johnston, G.M., 2011. The AUSGeoid2009 model of the Australian Height Datum, Journal of Geodesy (online first). doi: 10.1007/s00190-010-0422-2.

Haagmans., R., de Min, E., van Gelderen, M., 1993. Fast evaluation of convolution integrals on the sphere using 1D FFT, and a comparison with existing methods for Stokes' integral, manuscripta geodaetica 18(5): 227-241.

Hamming, R.W., 1986. Numerical methods for scientists and engineers, second edition. Reprint, Dover publications.

Heck, B., Grüninger, W., 1987. Modification of Stokes' integral formula by combining two classical approaches. Proceedings of the XIX General Assembly of the International Union of Geodesy and Geophysics, Vol. 2. Vancouver, Canada, pp. 309-337.

Heiskanen, W.A., Moritz, H., 1967. Physical geodesy. Freeman, San Francisco, 364 pages.

Hirt, C., Featherstone, W.E., Claessens, S.J., 2011. On the accurate evaluation of geodetic convolution integrals. Journal of Geodesy, submitted.

Holmes, S.A., Pavlis, N.K., 2008. Spherical harmonic synthesis software harmonic_synth. (Online at: http://earth-info.nga.mil/GandG/wgs84/gravitymod/egm2008/index.html).

Hotine, M., 1969. Mathematical Geodesy. ESSA Monograph No 2, US Department of Commerce, Washington DC.

Jekeli, C., Yanh, H.J., Kwon, J.H., 2009. Evaluation of EGM08 - globally and locally in South Korea. Newton’s Bulletin Issue n 4, April 2009 ISSN 1810-8555, Publication the International Association of Geodesy and International Gravity Field Service, pp 38-49.

Paul, M.K., 1973. A method of evaluating the truncation error coefficients for geoidal height. Bulletin Géodésique 47, 413-425.

Pavlis, N.K., Holmes, S.A., Kenyon, S.C., Factor, J.K., 2008. An Earth Gravitational Model to Degree 2160: EGM2008. Presented at the 2008 General Assembly of the European Geoscience Union, Vienna, Austria, April 13-18, 2008.

Press, W.H., Teukolsky, S.A., Vetterling, W.T., Flannary, B.P., 2002. Numerical Recipes in Fortran C - The Art of Scientific Computing, Second Edition. Cambridge University Press, Cambridge. 
Sjöberg, L.E., 1984. Least squares modification of Stokes's and Vening Meinesz's formulas by accounting for the truncation and potential coefficient errors. Manuscripta geodetica 9, 209-229.

Sjöberg, L.E., 1991. Refined least squares modification of Stokes’s formula. Manuscripta geodetica 16, 367-375.

Sjöberg, L.E. 2005. A discussion on the approximations made in the practical implementation of the remove-compute-restore technique in regional geoid modelling. Journal of Geodesy 78(11-12): 645-653.

Strang van Hees, G., 1990. Stokes formula using Fast Fourier Techniques. manuscripta geodaetica 15, 235-239.

Stark, P.A., 1970. Introduction to numerical methods. Macmillan, London, 334 pages.

Torge, W., 2001. Geodesy, third edition. De Gruyter, Berlin New York, 416 pages.

Vaníček, P., Kleusberg, A., 1987. The Canadian geoid - Stokesian approach. manuscripta geodaetica 12, 86-98.

Vaníček, P., Krakiwsky E.J., 1986. Geodesy: The concepts. Elsevier, Amsterdam New York, pp. 459-584.

Vaníček, P., Sjöberg, L.E., 1991. Reformulation of Stokes's theory for higher than seconddegree reference field and modification of integral kernels. Journal of Geophysical Research 96(B4), 6529-6539.

Vaníček, P., Featherstone, W.E., 1998. Performance of three types of Stokes’s kernel in the combined solution for the geoid. Journal of Geodesy, 72(12): 684-697, doi: $10.1007 / \mathrm{s} 001900050209$.

Wenzel, H.-G., 1982. Geoid computation by least squares spectral combination using integral kernels. Proceedings of the International Association of Geodesy General Meeting, Tokyo, Japan, pp 438-453.

Wessel, P., Smith, W.H.F., 1998. New, improved version of the Generic Mapping Tools released. EOS Trans. AGU 79, 579.

Wild-Pfeiffer, F., 2008. A comparison of different mass elements for use in gravity gradiometry. J Geod 82(10) 637-653, doi 10.1007/s00190-008-0219-8.

Wong, L., Gore, R., 1969. Accuracy of geoid heights from modified Stokes kernels. Geophysical Journal of the Royal Astronomical Society 18, 81-91.

\section{List of Figure captions:}



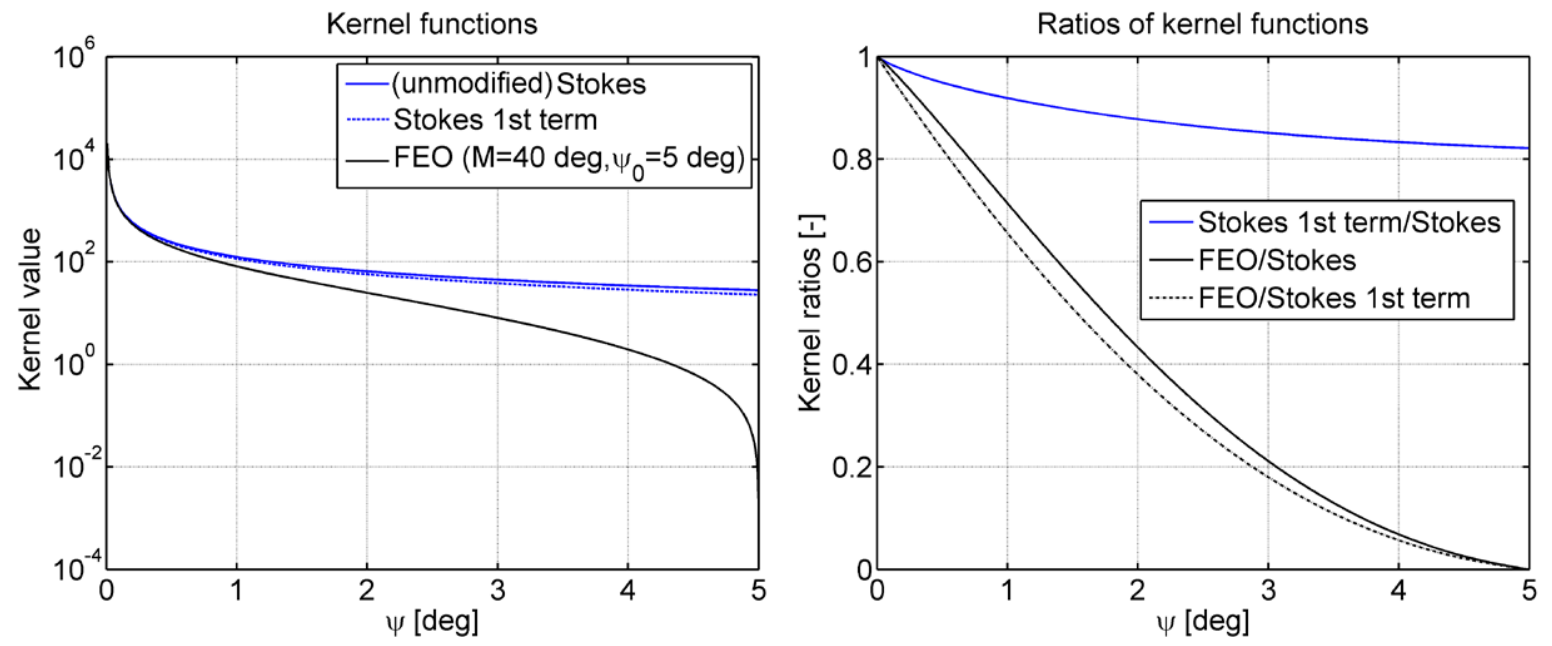

Fig. 1. Left: (unmodified) Stokes's function $S(\psi)$, first term of Stokes's function $S^{1 s t}(\psi)$ and FEO modified kernel $S_{F E O}(\psi)$ with $M=40$ and $\psi_{0}=5^{\circ}$. Right: Ratios $S^{1 s t}(\psi) / S(\psi)$, $S_{F E O}(\psi) / S(\psi)$ and $S_{F E O}(\psi) / S^{1 s t}(\psi)$.

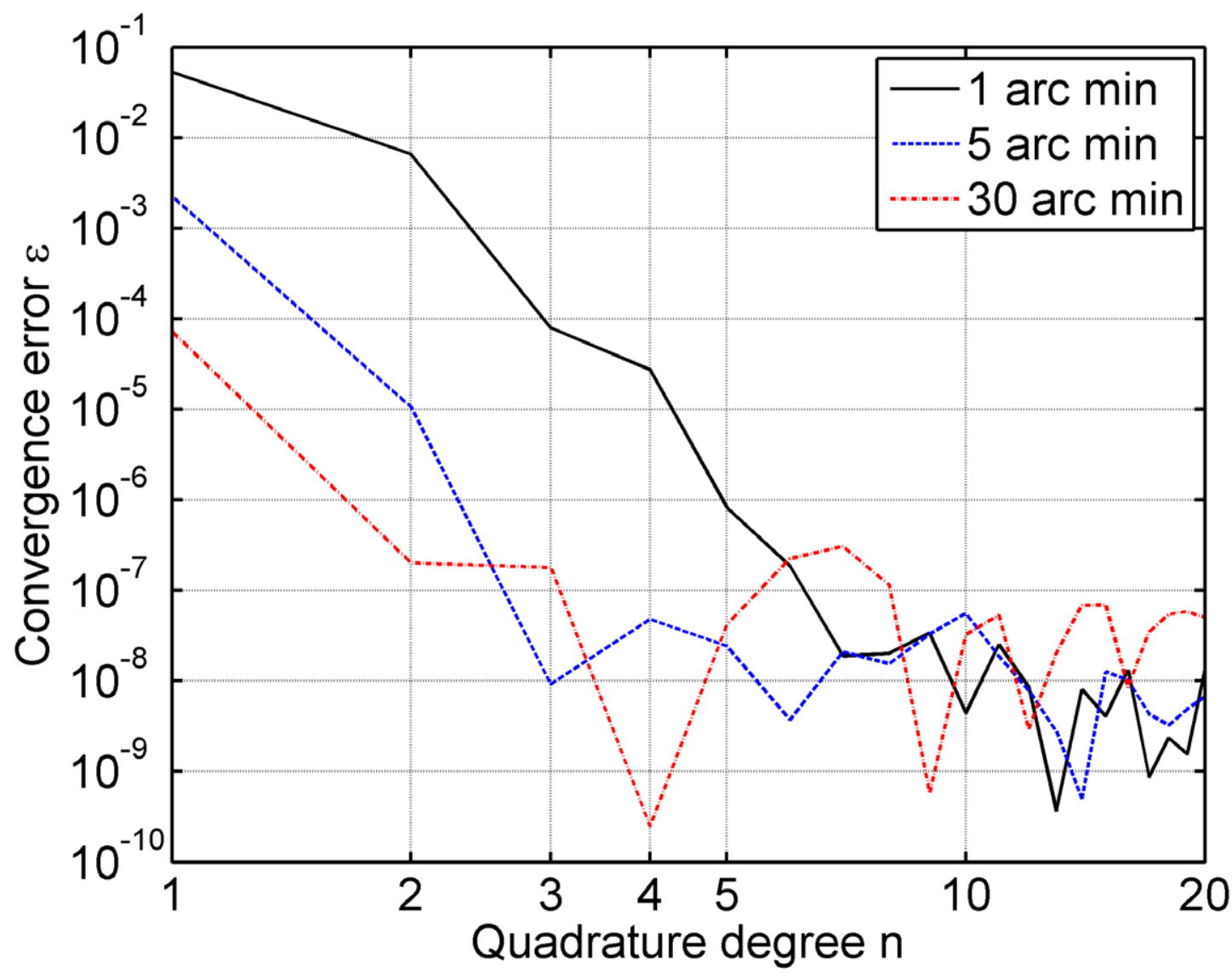


Fig. 2. Convergence error $\varepsilon$ of GLQ numerical mean kernels as a function of the quadrature degree $n$ and as a function of the distance $\psi$ between the innermost zone and the cell under evaluation (1, 5 and 30 arcmin). Cell resolution is 1 arc min $x 1$ arc min and latitude of the computation point $P$ is $-35^{\circ}$. Kernel is the FEO modification $\left(M=40, \psi_{0}=5 \mathrm{deg}\right)$.
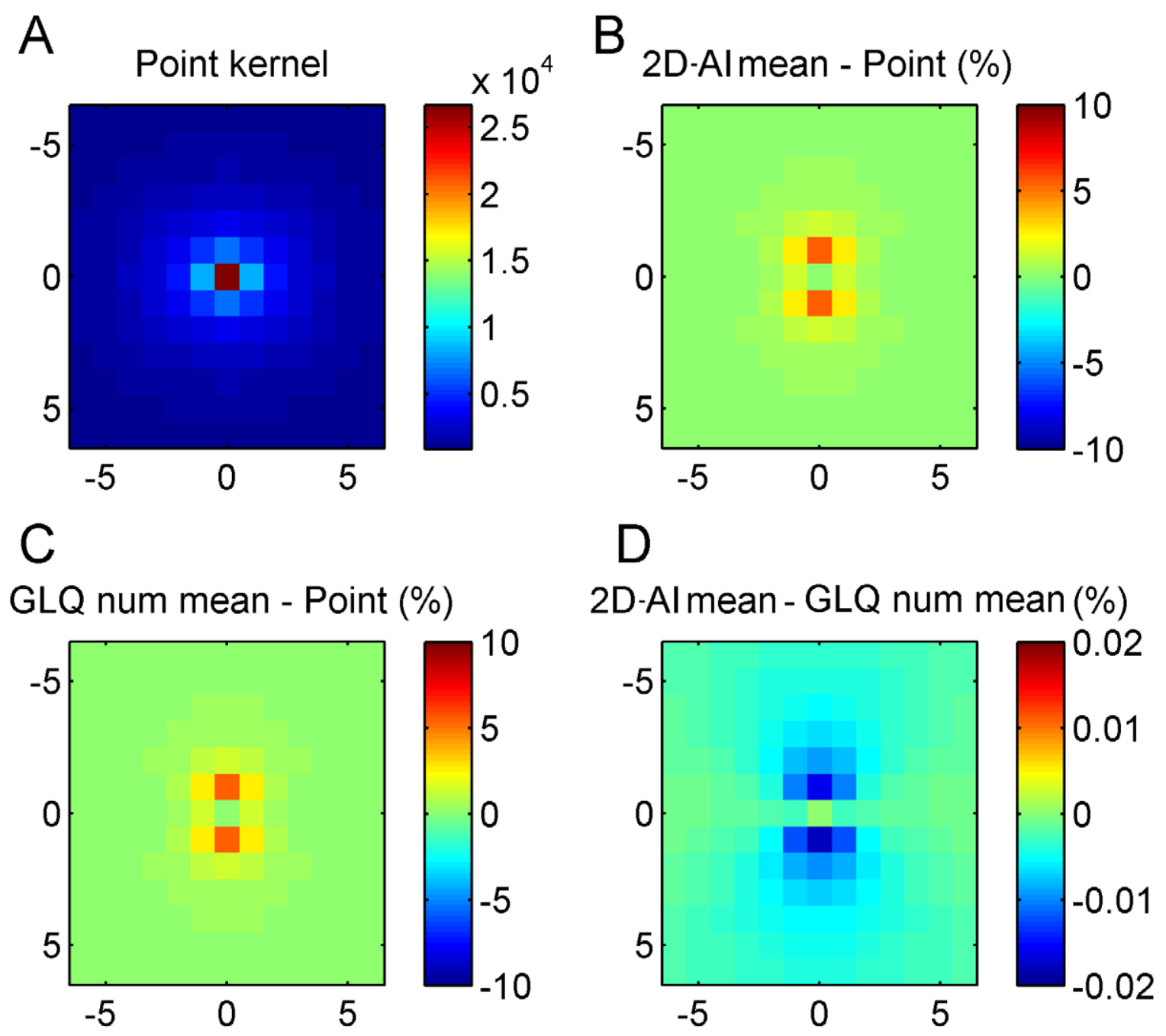

Fig. 3. A: Centre-of-cell (point) values of the FEO kernel. B: Percentage differences between FEO 2D-AI mean and point kernel. C: Percentage differences between GLQ numerical mean and point kernel. D: Percentage differences between 2D-AI and GLQ numerical mean kernel. The panels show $13 \times 13$ cell arrays with the centre being the computation point $P$. Cell resolution is 1 arc min $\times 1$ arc min and latitude of the computation point $P$ is $-35^{\circ}$. FEO kernel parameters are $M=40$ and $\psi_{0}=5$ deg. 


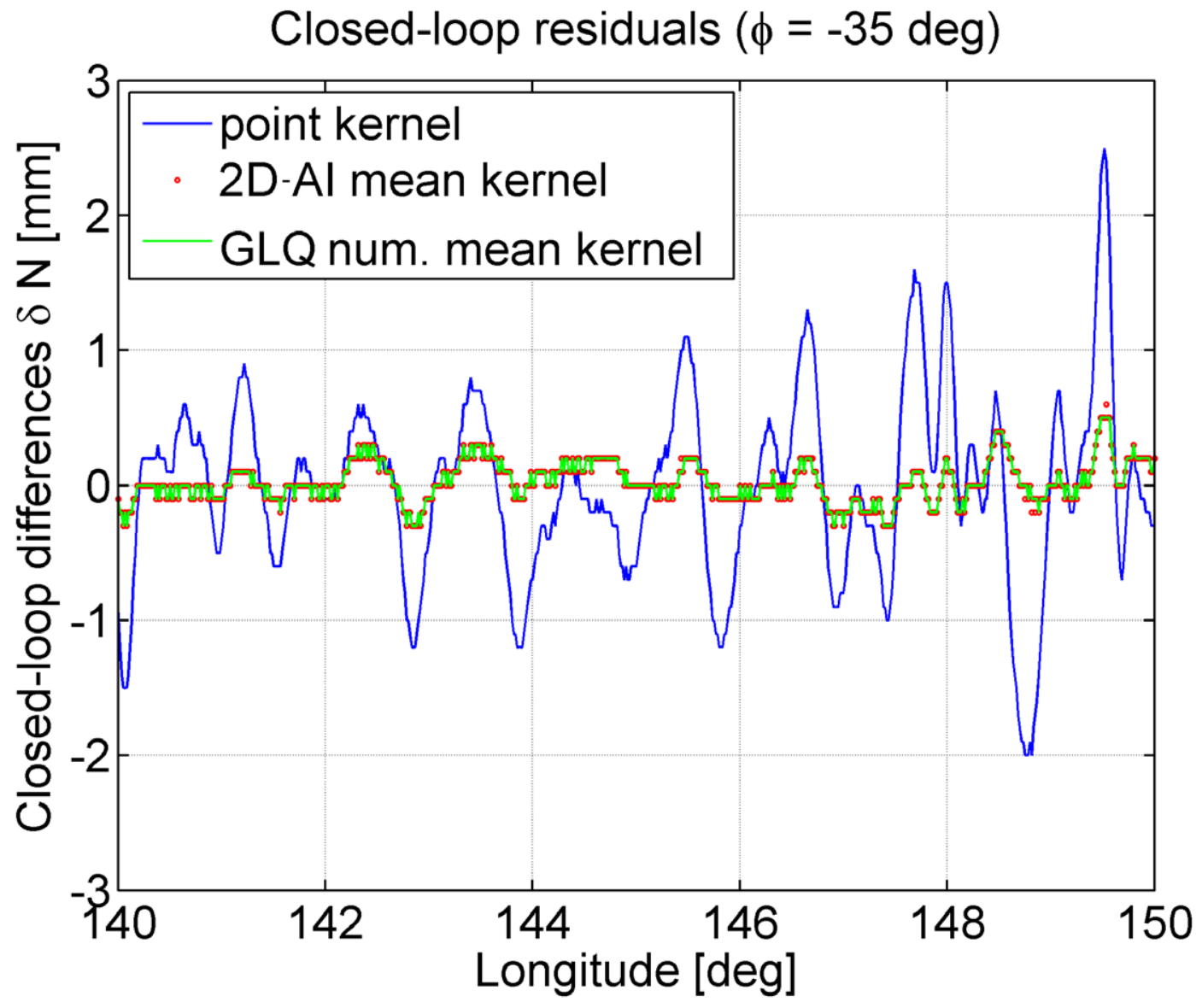

Fig. 4. Closed-loop differences $N_{E G M 2008}-N\left(\Delta g_{E G M ~ 2008}\right)$ over Australia along parallel -35 as a function of the FEO kernel variant (point, 2D-AI mean and GLQ numerical mean). 


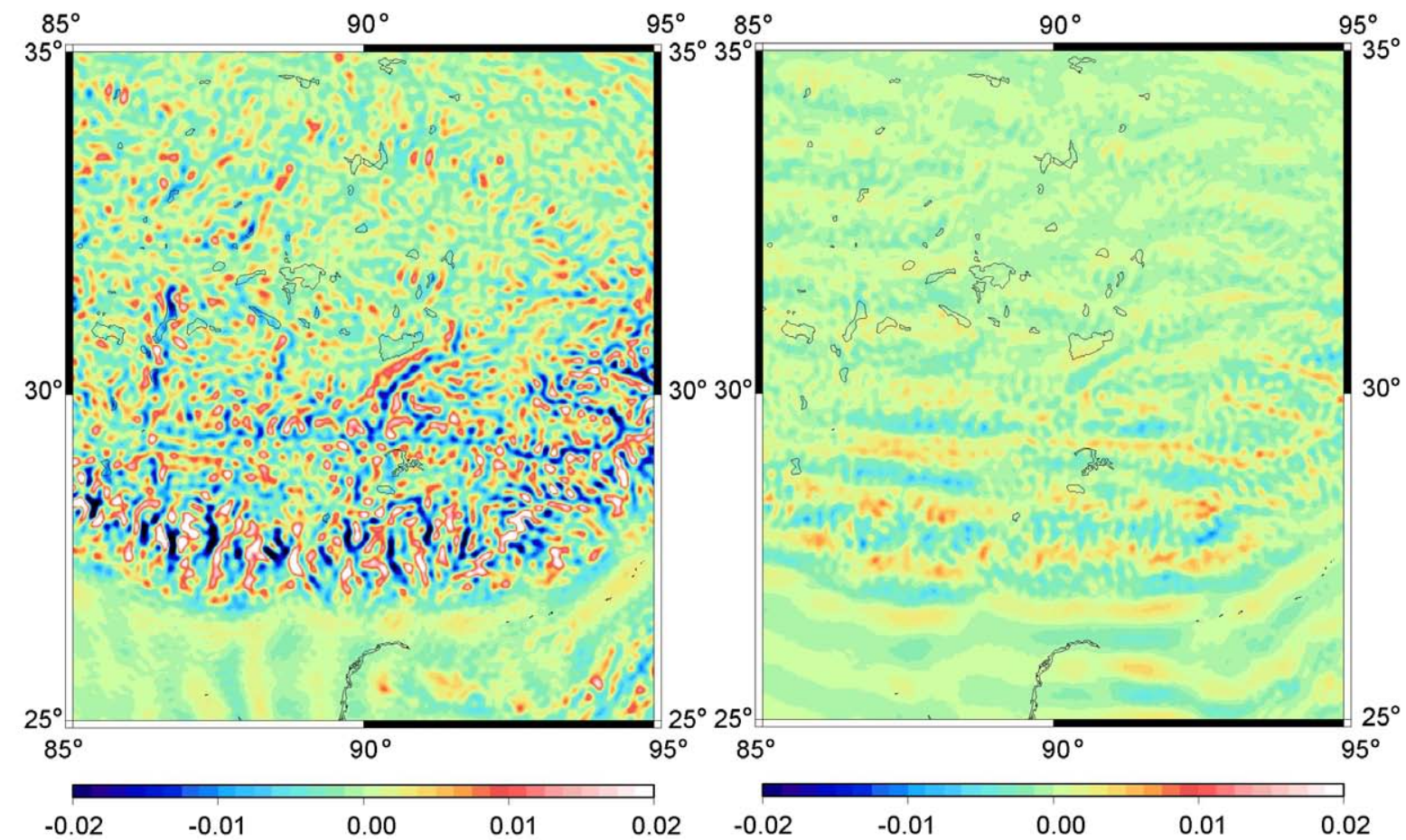

Fig. 5. Closed-loop differences $N_{E G M 2008}-N\left(\Delta g_{E G M ~ 2008}\right)$ for the Himalaya test area. Left: results of modified Stokes's integration using point kernels, Right: results of modified Stokes's integration using GLQ numerical mean kernels. Kernel used is the FEO modification ( $\left.M=40, \psi_{0}=5 \mathrm{deg}\right)$. Units in metres.

\section{Tables}

Table 1. Descriptive statistics of closed-loop geoid differences $N_{E G M 2008}-N\left(\Delta g_{E G M 2008}\right)$ for test area Australia $\left(-40^{\circ} \leq \varphi \leq-30^{\circ}\right.$ and $140^{\circ} \leq \lambda \leq 150^{\circ}$, 361201 pts, EGM2008 $361 . .2190$ truncation, $\left.N_{E G M 2008} \mathrm{~min} / \mathrm{max} / \mathrm{RMS}:-0.82 / 1.10 / 0.13 \mathrm{~m}\right)$. Units in mm.

\begin{tabular}{|l|l|l|l|l|}
\hline Kernel type & Min & Max & Mean & RMS \\
\hline Point ( centre-of-cell) & -9.0 & 14.3 & 0.0 & 1.2 \\
\hline 2D-AI analytical mean & -3.4 & 3.6 & 0.0 & 0.4 \\
\hline GLQ numerical mean & -3.1 & 3.0 & 0.0 & 0.4 \\
\hline
\end{tabular}

Table 2. Descriptive statistics of closed-loop geoid differences $N_{E G M ~ 2008}-N\left(\Delta g_{E G M ~ 2008}\right)$ for test area Europe $\left(35^{\circ} \leq \varphi \leq 55^{\circ}\right.$ and $5^{\circ} \leq \lambda \leq 25^{\circ}, 1442401$ pts, EGM2008 $361 . .2190$ truncation, $\left.N_{E G M 2008} \mathrm{~min} / \mathrm{max} / \mathrm{RMS}:-1.81 / 1.80 / 0.22 \mathrm{~m}\right)$. Units in mm. 


\begin{tabular}{|l|l|l|l|l|}
\hline Kernel type & Min & Max & Mean & RMS \\
\hline Point ( centre-of-cell) & -20.3 & 23.0 & 0.0 & 2.1 \\
\hline 2D-AI analytical mean & -8.0 & 8.3 & 0.0 & 0.8 \\
\hline GLQ numerical mean & 7.1 & 7.4 & 0.0 & 0.8 \\
\hline
\end{tabular}

Table 3. Descriptive statistics of closed-loop geoid differences $N_{E G M 2008}-N\left(\Delta g_{E G M 2008}\right)$ for test area Himalaya $\left(25^{\circ} \leq \varphi \leq 35^{\circ}\right.$ and $85^{\circ} \leq \lambda \leq 95^{\circ}$, 361201 pts, EGM2008 $361 . .2190$ truncation, $\left.N_{\text {EGM 2008 }} \mathrm{min} / \mathrm{max} / \mathrm{RMS}:-2.84 / 3.67 / 0.49 \mathrm{~m}\right)$. Units in mm.

\begin{tabular}{|l|l|l|l|l|}
\hline Kernel type & Min & Max & Mean & RMS \\
\hline Point ( centre-of-cell) & -35.7 & 37.8 & 0.0 & 5.2 \\
\hline 2D-AI analytical mean & -10.5 & 8.5 & 0.0 & 1.7 \\
\hline GLQ numerical mean & -11.3 & 9.0 & 0.0 & 1.8 \\
\hline
\end{tabular}

